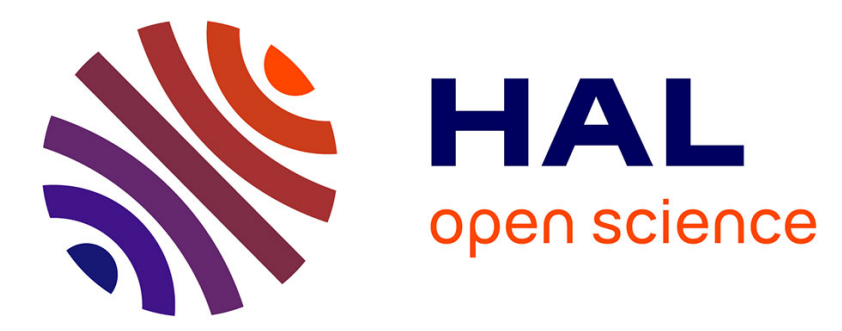

\title{
Quantification of the aggregation of magnetic nanoparticles with different polymeric coatings in cell culture medium
}

D Eberbeck, M Kettering, C Bergemann, P Zirpel, I Hilger, L Trahms

\section{- To cite this version:}

D Eberbeck, M Kettering, C Bergemann, P Zirpel, I Hilger, et al.. Quantification of the aggregation of magnetic nanoparticles with different polymeric coatings in cell culture medium. Journal of Physics D: Applied Physics, 2010, 43 (40), pp.405002. 10.1088/0022-3727/43/40/405002 . hal-00569546

\author{
HAL Id: hal-00569546 \\ https://hal.science/hal-00569546
}

Submitted on 25 Feb 2011

HAL is a multi-disciplinary open access archive for the deposit and dissemination of scientific research documents, whether they are published or not. The documents may come from teaching and research institutions in France or abroad, or from public or private research centers.
L'archive ouverte pluridisciplinaire HAL, est destinée au dépôt et à la diffusion de documents scientifiques de niveau recherche, publiés ou non, émanant des établissements d'enseignement et de recherche français ou étrangers, des laboratoires publics ou privés. 


\title{
Quantification of the aggregation of magnetic nanoparticles with different polymeric coatings in cell culture medium
}

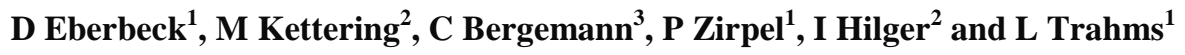 \\ E-mail: dietmar.eberbeck@ptb.de \\ ${ }^{1}$ Physikalisch-Technische Bundesanstalt, Abbestrasse 2-12, 10587 Berlin, Germany \\ ${ }^{2}$ Institute of Diagnostic and Interventional Radiology, University Hospital Jena, Erlanger Allee \\ 101, 07747 Jena, Germany \\ ${ }^{3}$ chemicell GmbH, Eresburgstraße 22-23, 12103 Berlin, Germany
}

\begin{abstract}
The knowledge of the physico-chemical characteristics of magnetic nanoparticles (MNPs) is essential to enhance the efficacy of MNP-based therapeutic treatments (e.g. magnetic heating, magnetic drug targeting). According to the literature, the MNP uptake by cells may depend on the coating of MNPs, the surrounding medium as well as on the aggregation behaviour of the MNPs. Therefore, in this study, the aggregation behaviour of MNPs in various media was investigated. MNPs with different coatings were suspended in cell culture medium (CCM) containing fetal calf serum (FCS), and the distribution of the hydrodynamic sizes was measured by magnetorelaxometry (MRX). FCS as well as BSA-buffer (phosphate buffered saline with $0.1 \%$ bovine serum albumin) may induce MNP aggregation. Its strength depends crucially on the type of coating. The degree of aggregation in CCM depends on its FCS content showing a clear, local maximum at FCS concentrations, where the IgGconcentration (part of FCS) is of the order of the MNP number concentration. Thus, we attribute the observed aggregation behaviour to the mechanism of agglutination of MNPs by serum compartments as for example IgG. No aggregation was induced for MNPs coated with dextran, polyarabic acid, or sodium phosphate, respectively, which were colloidally stable in CCM.
\end{abstract}

\section{Introduction}

Magnetic nanoparticles (MNPs) have been a research topic of great interest over the past 50 years (Gupta and Gupta 2005). Especially in the last decade, many novel applications of MNPs have been proposed and investigated for biological or medicinal approaches remarkably in the fields of Magnetofection $^{\text {TM }}$ (e.g. Mykhaylyk et al 2009), magnetic separation procedures (e.g. Clement et al. 2006), magnetic resonance imaging (MRI) as contrast agents (e.g. Sosnovik et al. 2008), magnetic drug targeting and delivery (e.g. Alexiou et al. 2007, Polyak et al. 2009) as well as in cancer treatments due to hyperthermal (e.g. Ito et al. 2003, Wilhelm et al. 2007, Thiesen\&Jordan 2008) or thermo ablative therapies (e.g. Hilger et al. 2001, Bruners et al. 2009).

Among the wide variety of MNPs, iron oxide (magnetit/maghemit) nanoparticles were and still are the most intensively studied MNPs (Lin et al. 2008, Peng et al. 2008). Obviously, this is due to the proven biocompatibility and the approval of superparamagnetic iron oxide nanoparticles (SPIOs) as contrast agents for MRI diagnostics in the clinics. Beside the necessity for biocompatibility studies with MNPs for medical applications, the further behaviour of MNPs in biological environments is relevant to be investigated. For in vivo applications, the knowledge of the aggregation behaviour of MNPs plays an important role, particularly for applications of MNPs into the vascular system for diagnostic or therapeutic purposes. Furthermore, for therapeutic cancer treatment due to magnetic 
heating, a high intracellular MNP loading is essential for the destruction of tumour cells (Kettering et al. 2007), in addition to a high specific heating power (SHP) (Hergt et al. 2006). To this end, it is essential to know which characteristics of MNPs increase their uptake in cell cultures. Here, the aggregation behaviour, in different surrounding media, in particular in cell cultures, plays an important role, as former studies showed (Tiefenhauer et al. 1996, Zhang et al. 2002, Gupta\&Gupta 2005, Sahoo\&Labhasetwar 2006). Also, the presence of FCS in the cell culture medium (CCM) is known to be of crucial importance (Chen et al. 2008, Petri-Fink et al. 2008) as well as the size of the nanoparticles (LaConte et al. 2005).

It was found for example that the efficiency of the uptake of gold nanoparticles or polymeric nanoparticles depends on their size (Sahoo\&Labhasetwar 2006, Chitrani et al. 2006, Win\&Feng 2005). Here, we will note for example that the parameters coating and MNP size seem to effect independently on the uptake (e.g. Petri-Fink et al. 2008). Furthermore, Dass et al. showed that nanoparticles were ingested by HeLa cells in endosomes both individually (one per endosome) and in form of aggregates (several per endosome), depending on the composition of nanoparticles (Dass et al. 2007).

In this context, the aim of the presented work was to examine the aggregation behaviour of superparamagnetic iron oxide nanoparticle formulations differing in their coatings in relevant media. The knowledge about their aggregation behaviour is important to estimate and understand different internalization amounts into cells with regard to therapeutic approaches like magnetic heating treatments [Hilger 2005] or magnetic drug targeting (Alexiou et al. 2007). To this end, the impact of coating and medium on the effective particle size can be evaluated, which is one parameter that determines the uptake process into target cells,. The aggregation behaviour in dependence of the various biocompatible coatings should be investigated in CCM to elucidate the in vitro situation for tests with cultured cell lines and in BSA-buffer (phosphate buffered saline (PBS) with $0.1 \%$ bovine serum albumin (BSA)) as a control medium.

Because of their long blood circulation time, MNPs with hydrophilic polymer coatings like dextran or polyethylene glycol (PEG) (Gupta\&Gupta 2005) are of particular interest with respect to further in vivo applications. In our own MRX studies, we found that MNPs with hydrophilic electrically charged coatings may aggregate in FCS and human serum, while those with non-charged starch layer do not (Eberbeck et al. 2005, Eberbeck et al. 2006). In order to keep colloidal stability in salty media, we chose 12 different formulations of superparamagnetic iron oxide nanoparticles with highly hydrophilic polymer coatings. In order to investigate the effect of its electrical charge coatings with neutral $(\mathrm{OH}-$ groups), negatively (e.g. $\mathrm{COOH}-$ ) and positively charged (NH-) groups were chosen. Different negatively charged functional groups were applied in order to disentangle the influences of the charge and the nature of the functional groups.

It was found, that the uptake of MNPs depends on the amount of incubated MNPs (Petri-Fink et al. 2005). In particular, the aggregation of MNPs in FCS containing media depends on the MNP-to-serum concentration ratio, where the mechanism of agglutination of MNPs (Eberbeck et al. 2009) caused by particular FCS-proteins, e.g. antibodies, is discussed (Eberbeck et al. 2005, Eberbeck et al. 2006). Therefore, within this study, we will address the concentration dependence of the aggregation of MNPs in CCM.

Because of their high performance in Magnetofection ${ }^{\mathrm{TM}}$, we investigated multicore MNPs with hydrodynamic diameters equal or larger than $100 \mathrm{~nm}$. Also the contrast agent Resovist ${ }^{\circledR}$ was investigated as a reference system, because it is already approved for the clinical application and because its colloidal behaviour was already known from our earlier MRX studies (Eberbeck et al. 2005, Eberbeck et al. 2006) . 
In the present study we again applied MRX which is allows for a very robust measurement of MNPs in non transparent media like blood (Eberbeck et al. 2009). In contrast to the PCS-method (photon correlation spectroscopy), frequently used for the estimation of aggregate size, MRX signals are not affected by other (non magnetic) corpuscular compartments like cells or other particulate impurities which may strongly interfere PCS measurements.

The present study is based on these former studies to deepen the knowledge of the aggregation behaviour of MNPs for further in vitro and in vivo investigations. In this study, we focus on the in vitro aggregation behaviour of MNP in dependence on the biocompatible coating with different functional groups.

\section{Experimental}

\subsection{Materials}

Bovine serum albumin buffer (BSA-buffer: phosphate buffered saline (PBS) containing $138 \mathrm{mmol} \mathrm{l}^{-1} \mathrm{NaCl}, 2.7 \mathrm{mmol}^{-1} \mathrm{KCl}$, and $10 \mathrm{mM}$ phosphate with $0.1 \%$ bovine serum albumin) was used for the dilution of MNP stock suspensions and for reference samples. The albumin concentration is $0.1 \%$ by weight, i.e. $1.5 \times 10^{-5} \mathrm{~mol}^{-1}$. The medium of our interest is a cell culture medium (CCM) composed of Dulbecco's Modified Eagle Medium with F-12 Nutrient Mixture (D-MEM/F-12, \#11320-074, Invitrogen, Karlsruhe, Germany) containing $1 \%$ or $10 \%(\mathrm{v} / \mathrm{v})$ foetal calf serum (FCS, \#10270, Invitrogen, Karlsruhe, Germany), corresponding to a total albumin concentration of $0.02 \%$ and $0.2 \%$ by weight. Furthermore, the FCS-concentration in the CCM was varied within the range $0.022 \%$ and $67 \%$. The concentration of $\gamma$-immunoglobulins within the FCS stock solution was $\beta(\gamma$-immunoglobulin $)=126.5 \mathrm{mg} / \mathrm{l}$. Most of the $\gamma$-immunoglobulins are antibodies being exclusively IgG antibodies in FCS. Thus, the IgG concentration was roughly estimated to be $c(\gamma$-immunoglobulin $)=843 \mathrm{nmol} / 1 \approx c(\operatorname{IgG})$.

Magnetic nanoparticles (chemicell $\mathrm{GmbH}$, Berlin, Germany) are obtained by converting an acidic iron(II/III) salt solution into iron(II/III)-carbonate by adding equivalent amounts of alkaline carbonate, followed by a successive thermic reaction to iron(II,III) oxide according to a recently described method (Bergemann 1996). After removing of surplus salts, the particles were coated with water soluble polysaccharides or polymers. The 12 investigated MNP formulations mainly differ in its coatings, ranging from electrically nearly neutral dextran and starch over negatively charged carboxymethyl dextran, polygalacturonic acid, polyarabic acid, phosphorylated starch, dextransulphate, heparin, oleic acid to positively charged diethylaminoethyl and polyethylenimine the properties of which are listed in table 1. In contrast to the fluidMAGs, the starch matrix of targetMAG-NC is additionally cross linked and modified with terminal phosphate groups.

For comparison, we also measured the well known commercially available MRI contrast enhancing agent Resovist ${ }^{\circledR}$ which comprises magnetite cores, where single cores and small clusters (mean hydrodynamic size is $60 \mathrm{~nm}$ ) are coated with carboxymethyl dextran (table 1; Bayer Health Care, Berlin. Germany). In earlier investigations (Eberbeck et al. 2006) the effective (cluster!) core size distribution with a mean diameter of about $8.5 \mathrm{~nm}$ was deduced from MRX-measurements. The corresponding diameter of the mean core volume is about $d_{\mathrm{V}}=10 \mathrm{~nm}$.

\subsection{MRX-measurements}

For the measurement of the magnetic relaxation, a sample of $140 \mu 1$ volume (within a PE micro vial) was placed in the $10 \mathrm{~mm}$ bore of the magnetizing coil. A magnetic field of $1.3 \mathrm{kA} / \mathrm{m}$ was applied for $1 \mathrm{~s}$ to align the magnetic moments within the sample. After jump-likely switching off the field within about $500 \mu$ s, the decay of the magnetic field in z-direction was measured by a low-Tc SQUID 
at a distance of $10 \mathrm{~mm}$ above the sample. The device and the method were described in detail in (Matz et al. 1999). The measurement time window was $450 \mu \mathrm{s} \leq t \leq 0.45 \mathrm{~s}$. The output signal of SQUID sensor contained an unknown offset value $B_{\text {offset }}$. For visual comparison purposes, the offset of each measured relaxation curve $B(t)$ was adjusted so that all relaxation curves coincided at the end of the measurement.

The relaxation curves could be characterized by two main parameters, the relaxation amplitude $\Delta B=B(0.61 \mathrm{~ms})-B(350 \mathrm{~ms})$ and the relaxation time $t_{1 / \mathrm{e}}$ which is the time where $B\left(t_{1 / \mathrm{e}}\right)-$ $B(350 \mathrm{~ms})=1 / \mathrm{e} \Delta B$ counted from the reference point of $0.61 \mathrm{~ms}$.

It was previously shown, that from the relaxation curves the distribution of the sizes of hydrodynamic entities - in our case the MNPs including their solvatisation shell as well as clusters of MNPs again with their solvatisation shells - can be extracted. For that purpose, the so called cluster moment superposition model (CMSM) (Eberbeck et al. 2005)

$$
\begin{aligned}
B(t)= & g M_{\mathrm{S}} \int_{d} f(\mu, \sigma, d) L\left(M_{\mathrm{S}}, d, T, H\right) A_{\mathrm{C}} \int_{d_{\mathrm{C}}} f_{\mathrm{C}}\left(\mu_{\mathrm{C}}, \sigma_{\mathrm{C}}, d_{\mathrm{C}}\right) \times \\
& \times\left(1-\exp \left\{-\frac{t_{\mathrm{mag}}}{\tau_{\text {eff }}\left(d, d_{\mathrm{C}}, M_{\mathrm{S}}, K_{\text {eff }}, \eta, H, T\right)}\right\}\right) \exp \left\{-\frac{t}{\tau_{\text {eff }}\left(d, d_{\mathrm{C}}, K_{\text {eff }}, \eta, T\right)}\right\} \mathrm{d} d_{\mathrm{C}} \mathrm{d} d
\end{aligned}
$$

was fitted to the MRX-curves. Here, $g$ is an amplitude factor determined by the measurement geometry, $M_{\mathrm{S}}$ is the saturation magnetisation of the cores, $f$ is the distribution of the core diameters $d$ with the median size $\mu$ and the size distribution parameter $\sigma, L$ is the Langevin function depending on $d$, the magnetising field $H$, the magnetising time $t_{\text {mag }}$, and the temperature $T ; A_{\mathrm{C}}$ is the fraction of MNPs belonging to aggregates, here modelled by spherical clusters with a distribution $f_{\mathrm{C}}$ of the cluster diameters $d_{\mathrm{C}}$ determined by the corresponding median and distribution parameter $\mu_{\mathrm{C}}$ and $\sigma_{\mathrm{C}}$, and finally $\tau_{\text {eff }}$ was the effective relaxation time according to $\tau_{\text {eff }}^{-1}=\tau_{\mathrm{N}}^{-1}+\tau_{\mathrm{B}}^{-1}$ where $\tau_{\mathrm{N}}$ and $\tau_{\mathrm{B}}$ are the Néel and Brownian relaxation times of the particle moments. From $f_{\mathrm{C}}$,

$$
f(\mu, \sigma, d)=\frac{1}{\sqrt{2 \pi} \sigma d} \exp \left\{-\frac{\ln (d)-\ln (\mu)}{2 \sigma^{2}}\right\}
$$

we calculated the diameter of the cluster with the mean volume $d_{\mathrm{CV}}$ and the mean cluster diameter from the volume weighted cluster diameter distribution function according to $d_{\mathrm{CwV}}=\int d_{\mathrm{C}} d_{\mathrm{C}}^{3} f\left(d_{\mathrm{C}}\right) \mathrm{d} d_{\mathrm{C}} / \int d_{\mathrm{C}}^{3} f\left(d_{\mathrm{C}}\right) \mathrm{d} d_{\mathrm{C}}$. This average regarded the sample volume occupied by the clusters as weight.

\section{Results}

The aggregation of MNPs was quantified from MRX data measured shortly (about 90 minutes) after preparation. Here, the dispersion parameter of the cluster size distribution, obtained from model fit, has proved to be a suitable parameter for classification of the aggregation. These results were compared with the degree of precipitation, visually observed about 19 hours after preparation, classified in "no", "weak", "medium" and "strong" precipitation (figure 1). On this basis we classified and quantified the aggregation of the different MNPs, suspended in the cell culture medium (CCM) and in BSA-buffer as a reference.

\subsection{Aggregate size distribution}

Fitting the cluster moment superposition model (CMSM) to the relaxation curves, we got the distribution of the hydrodynamic sizes of the relaxing entities, i.e. MNPs and aggregates of them, further on denoted as cluster- or aggregate size distribution. In order to interpret the results for the 
cluster size distribution, we considered first the data obtained for Resovist ${ }^{\circledR}$ where the single cores particles had a mean core diameter of around $4 \mathrm{~nm}$ coated with a carboxydextran layer with a mean thickness of about $4 \mathrm{~nm}$ (Lawaczeck et al. 1997). In BSA buffer, the mean hydrodynamic volume corresponded to a cluster diameter of $d_{\mathrm{CV}}=47 \mathrm{~nm}$ in good agreement with the data of a previous study (Eberbeck 2005) and with PCS data, $d_{\mathrm{PCS}}=61 \mathrm{~nm}$ (Eberbeck et al. 2006) (table 2). In BSA-buffered suspension, we got a moderate width of the cluster size distribution of Resovist ${ }^{\circledR}$ MNPs indicated by the distribution parameter $\sigma_{\mathrm{C}}=0.54$. In contrast, if Resovist ${ }^{\circledR}$ was suspended in the CCM (containing FCS), $\sigma_{\mathrm{C}}$ was rising to the large value of 1 (table 2). The values of mean aggregate size of $d_{\mathrm{CV}}=3 \mathrm{~nm}$ and $8 \mathrm{~nm}$ were not meaningful, since these values were smaller than the mean diameter of the coreshell particle being about $10 \mathrm{~nm}+8 \mathrm{~nm}=18 \mathrm{~nm}$. Also for the other MNP samples with strong visual precipitation, high $\sigma_{\mathrm{C}}$ values and a mismatch between mean aggregate sizes estimated by MRX, $d_{\mathrm{CV}}$, and PCS, $d_{\mathrm{PCS}}$, respectively, were observed, except for fluidMAG-PGS suspended in BSA-buffer (see example below). Vice versa, if the apparent cluster size distribution was narrow, indicated by low $\sigma_{\mathrm{C}}$ values $\left(\sigma_{\mathrm{C}}<0.6\right)$, then the MNP suspensions were visually long term stable in BSA buffer and $d_{\mathrm{Cv}}$ and $d_{\mathrm{PCS}}$ agreed well (table 2). This preliminary conclusion (working hypothesis) obtained from the measurements on Resovist ${ }^{\circledR}$ will be further illustrated by the following two examples concerning fluidMAG MNPs:

(i) The shape of the MRX curves of strongly aggregated MNPs (for example fluidMAG-Heparin in CCM with $1 \%$ FCS) was very similar to that of immobilised MNPs while those containing smaller (fluidMAG-ARA, $\quad d_{\mathrm{CwV}}=290 \mathrm{~nm}$ ) and even larger but well suspended (targetMAG-NC, $d_{\mathrm{CwV}}=597 \mathrm{~nm}$ ) aggregates distinguished in shape significantly from the curve of immobilised MNPs (figure 2). The CMSM analysis of the MRX curves of immobilised MNPs yields for all MNP formulations large $\sigma_{\mathrm{C}}$-values, e.g. $\sigma_{\mathrm{C}}=1.74$, and very small $d_{\mathrm{CV}}$-values, e.g. $d_{\mathrm{CV}}=14.4 \mathrm{~nm}$ (fluidMAG-ARA). Hence, we associated large values of $\sigma_{\mathrm{C}}$ with well extended aggregates, where the Brownian relaxation of the MNPs was strongly suppressed. In this case, the assumptions of the CMSM, i.e. the lognormal size distribution of clusters was obviously not valid. Consequently, the values of $d_{\mathrm{CV}}$ and $d_{\mathrm{CwV}}$ lost the physical meaning.

(ii) In contrast to the other fluidMAGs with strong visual aggregation, where large values of the dispersion parameter $\sigma_{\mathrm{C}}>0.84$ were observed, the strong aggregation in fluidMAG-DXS suspended in BSA buffer was accompanied by a relatively small value of $\sigma_{\mathrm{C}}=0.45$. Obviously, the aggregate structure of this sample distinguished qualitatively well from that of the other MNP formulations. This seems to correlate with the rather smooth appearance of the precipitate observed in fluidMAG-DXS, compared to that of mentioned other ones, where the appearance of the precipitates had a stronger contrast to the suspension medium.

In conclusion, if $\sigma_{\mathrm{C}}$ is small then $f_{\mathrm{C}}$ seems to obey a monomodal size distribution like (2) and (1) is applicable. But, if the estimated value for $\sigma_{\mathrm{C}}$ exceeds a value of about 1 , there are very large aggregates present, the magnetisation of which behaves like that of immobilised MNPs (within our measurement time window). Therefore, we used the distribution parameter $\sigma_{\mathrm{C}}$ as a tool for the assessment of the type of aggregates. In this latter case, the validity of (2) is questionable and the size distribution parameters $\mu_{\mathrm{C}}$ and $\sigma_{\mathrm{C}}$ serves only comparative purposes.

\subsection{Aggregation in BSA-buffer}

While all MNP formulations were stable in its original suspension medium (deionised water) some of them aggregated already in BSA buffer. First of all, fluidMAG-DEAE and -PEI aggregated immediately after suspension in BSA buffer, but also in pure PBS i.e. without BSA and in CCM 
without FCS. Because of this visually observed strong aggregation features, these samples were excluded from further investigations by MRX.

The relaxation behaviour of the different MNPs was measured about 90 minutes after preparation. At this time, no clear visual markers of aggregation in the suspensions were found. Only after 19 hours distinct precipitation was visually observed (figure 1 and table 2).

According to the tendency of particle aggregation in BSA buffer, we divided the different MNP formulations into 2 main groups: MNPs which are (i) colloidally stable or (ii) unstable in BSA-buffer, respectively. While the samples of group (i) were long term stable in BSA-buffer, i.e. they did not show any visually discernible precipitation after 19 hours, those of group (ii) showed at least weak precipitation (table 2). Within the group (i) there were 2 subgroups: The formulations fluidMAG-DX, -D, -CMX, -PGS showed low $\sigma_{\mathrm{C}}$ values $\left(\sigma_{\mathrm{C}}<0.6\right)$ and the mean aggregate sizes estimated by MRX, $d_{\mathrm{CV}}$, and PCS, $d_{\mathrm{PCS}}$, respectively, matched within an accuracy of about $30 \%$ (table 2 ). The $\sigma_{\mathrm{C}}$-values of the second subgroup (fluidMAG-ARA, fluidMAG-DP and targetMAG-NC) amounted to $0.72 \leq \sigma_{\mathrm{C}} \leq 0.84$ and the MRX and PCS sizes differed by about $80 \%$ (table 2). The formulations of group (ii) fluidMAG-DXS, fluidMAG-Heparin, and fluidMAG-OS showed high $\sigma_{\mathrm{C}}$ values, $\sigma_{\mathrm{C}} \geq 1$, except for fluidMAG-DXS, and the aggregate sizes estimated by MRX and PCS, respectively, differed strongly (table 2).

\subsection{Aggregation in cell culture medium with FCS}

The relaxation curves of fluidMAG-DX, fluidMAG-D, fluidMAG-ARA, and targetMAG-NC suspended in CCM containing $1 \%$ and $10 \%$ FCS, respectively, did not or only slightly distinguish from those of the samples suspended in BSA buffer, indicated by nearly the same values for $\sigma_{\mathrm{C}}, d_{\mathrm{CwV}}$, and $d_{\mathrm{PCS}}$, practically independent on the FCS concentration (table 2). The absence of strong visually observable precipitation after 19 hours (table 2) also indicated the (long term) coloidal stability of these MNP formulations.

All other MNP formulations aggregated strongly in 1\% FCS. Interestingly, the precipitation of the MNP formulations fluidMAG-DP, -DXS, and -Heparin was weaker in CCM with 10\% FCS than with $1 \%$ FCS. In accordance to the above outlined relation, this comparatively weaker aggregation corresponded to $\sigma_{\mathrm{C}^{-}}$-values, all around 0.64 , being much smaller than those for the strongly precipitating samples, where $\sigma_{\mathrm{C}}$ was $\geq 0.9$ (table 2 ).

In order to study the effect of the FCS concentration on the aggregation more thoroughly, we suspended the ferrofluids fluidMAG-DX, fluidMAG-D, fluidMAG-ARA and targetMAG-NC as well as Resovist ${ }^{\circledR}$ in FCS and in CCM containing different concentrations of FCS. First, we diluted the MNP suspensions in FCS-free CCM down to an iron concentration of about $4.8 \mathrm{mmol} / \mathrm{l}$. Then, $50 \mu \mathrm{l}$ MNP suspension were added to $100 \mu$ FCS-CCM- with a FCS volume fraction ranging from $100 \%$ to $1 \%$. So we could achieve higher FCS-to-MNP concentration ratios than in that experiments the results of which are presented in table 2. The relaxation curves for the formulations fluidMAG-ARA, fluidMAG-D, fluidMAG-DX and targetMAG-NC did not depend on the FCS concentration (figure 3) supporting the claim that they were colloidal stable in FCS. For fluidMAG-D there was, however, a weak violation of the matching of the relaxation curves at the FCS concentrations of $6.7 \%$ and $22 \%$ (figure 4). Note, that this behaviour was not caused by the slightly higher viscosity due to the higher FCS content, since this effect was absent in fluidMAG-DX, fluidMAG-ARA and targetMAG-NC (figure 3). In contrast, the curves of Resovist ${ }^{\circledR}$ samples changed smoothly in shape with respect to the FCS concentration as shown in figure 5 . The relaxation curve shape parameters $t_{1 / \mathrm{e}}$ and the extracted $\sigma_{\mathrm{C}}$ patterned smooth maxima at a FCS concentration corresponding to an IgG concentration of about $60 \mathrm{nmol} / \mathrm{l}$ (figure 3). With given iron concentration and mean particle core volume (being equivalent 
to a core diameter of $10 \mathrm{~nm}$ ), for Resovist $^{\circledR}$ we calculated a MNP concentration of $c(\mathrm{MNP})=80 \mathrm{nmol} / \mathrm{l}$. matching well with the concentration of $\operatorname{IgG}$ at the aggregation maximum. This finding agreed well with previous data obtained on basically the same MNP formulation of DDM128 (Meito Sangyo, Japan) (Eberbeck et al. 2006). The matching of the concentrations of MNPs and IgG at aggregation maximum was interpreted by agglutination of the MNPs by means of the $\operatorname{IgG}$ molecules (Eberbeck et al. 2006).

In order to check the long term aggregation stability, we measured the FCS concentration dependency of the extent of aggregation 17 hours after preparation of fluidMAG-D, fluidMAG-DX, fluidMAG-ARA and targetMAG-NC. The relaxation curves for fluidMAG-DX did not show any significant change and those of fluidMAG-ARA and targetMAG-NC indicated a change of hydrodynamic volume, i.e. apparent cluster volume, of about $10 \%$, only. On the other hand, the relaxation curves of fluidMAG-D changed dramatically (figure 5). Here, a FCS (or IgG) concentration dependent aggregation emerged very clearly after 17 hours, where the aggregation showed a local maximum similar to that of the Resovist ${ }^{\circledR}$ sample after 3 hours (figure 3). Nearly the same result was retrieved for a 10 times lower MNP concentration. Hereby, the position of the aggregation maximum was dependent first and foremost on the MNP-to-FCS concentration ratio. The different shape of this maximum may be associated with reduced aggregation kinetics in the more diluted sample due to the lower concentrations of MNPs and FCS. Assuming a mean core volume corresponding to $12 \mathrm{~nm}$, we got a MNP concentration of about $100 \mathrm{nmol} / 1$ and $10 \mathrm{nmol} / \mathrm{l}$ for the more diluted sample, respectively, matching well the $\operatorname{IgG}$ concentrations at the maximum position (figure 6). Hence, we infer that agglutination took place in fluidMAG-D sample, which was very similar to that in Resovist ${ }^{\circledR}$, but with a much longer time constant.

We conclude, that the aggregation of Resovist ${ }^{\circledR}$, fluidMAG-D, -DP, -DXS, -Heparin, and -OS in CCM is very likely, at least partially, caused by an agglutination process mediated by IgG or other components (except albumin) of FCS. This interpretation was supported by the finding that the aggregation had its maximum where the concentrations of MNPs and IgG matched (Figures 3 and 6, (Eberbeck 2006)) and by the observation that the aggregation was much stronger in CCM with $1 \%$ FCS than with $10 \%$ FCS (table 2).

\section{Discussion}

On the base of the presented results, the analysed MNPs could be described as being (i) colloidally not stable in BSA-buffer and cell culture medium (CCM), (ii) colloidally stable, and (iii) exhibit agglutination behaviour in CCM.

(i) The MNP formulations fluidMAG-DEAE, -PEI and -DXS were colloidally not stable in BSA buffer and CCM. fluidMAG-DEAE and -PEI, having a highly positive zeta potential of about $50 \mathrm{mV}$ in deionised water, showed the strongest aggregation behaviour which was induced by an ion concentration of $140 \mathrm{mmol} / \mathrm{l}$ (PBS). Also, fluidMAG-DXS with a negative zeta potential showed a strong aggregation behaviour in BSA buffer. We attributed these aggregations to the known effect of the screening of electrostatic repulsive potential by ions (Sonntag\&Strenge 1987) in the suspension medium. It remained the steric repulsion between the MNPs, mediated by the DEAE, PEI and DXS shells, which obviously could not prevent the aggregation in the given case. Thus, in media with high ionic strength the shell thickness was not dense and/or thick enough to compensate the attractive forces generated by van der Waals and magnetic dipolar interaction. For example, it was shown in Eberbeck et al. (2006) that the total interaction potential of two electrically non-charged magnetite MNPs being in contact, having a core diameter of $15 \mathrm{~nm}$ and a thickness of the coating of $4 \mathrm{~nm}$ (reasonably parameters for the carboxydextran shell of Resovist ${ }^{\circledR}$ ), amounted to $-650 \mathrm{~K}$ at the equilibrium distance, i.e. the interaction potential has a highly attractive effect at room temperature 
( $T=295 \mathrm{~K}$ ). Furthermore, a coating consisting of hydrophilic polymer chains contained water. So, note that a high ion concentration may dehydrate the coating partially, presumably leading to its shrinkage which in turn reduces the particle-particle contact distance. Further in vitro studies with cell cultures should show whether these agglutinated particles could be internalized from cells more effectively due to an enlarged internalisation rate or an enhanced magnetically induced pressure of sedimentation. An additional interesting question would be if these particles have a distended cytotoxicity in contrast to particles that do not agglomerate in physiological media. For in vivo purposes, the injection of fluidMAG-DEAE, and -PEI to the vascular system is precarious because of the enhanced risk of vascular obliterations. In contrast, those particles might be suitable for magnetic heating treatments if they are injected directly into target regions and if they have appropriate specific absorption rates to generate enough heat for destroying target cells.

(ii) Long term colloidal stability in both CCM containing FCS and BSA buffer could be detected for fluidMAG-ARA, -DX and targetMAG-NC. Also fluidMAG-D was colloidally stable at room temperature at least for 90 minutes. The high colloidal stability of fluidMAG-DX and $-\mathrm{D}$ correlates well with low zeta potentials, this interrelation was already suggested in (Eberbeck et al. 2006). However, also fluidMAG-ARA and targetMAG-NC are colloidally stable, though they have a significant electrical surface potential due to carboxylic and phosphate functional groups, respectively. Consequently, fluidMAG-ARA, -D and -DX as well as targetMAG-NC seems to be harmless regarding agglomeration and possible vascular obliterations for in vivo purposes. Additional studies with those MNPs in whole blood should be done to confirm these findings.

(iii) On the other hand, the MNP suspensions of Resovist ${ }^{\circledR}$, fluidMAG-CMX, -PGS, -DP, -Heparin and -OS exhibited agglutination behaviour only in CCM. They aggregated partially already about 90 minutes after being transferred into CCM. Obviously, this was caused by those CCM components which are not part of BSA buffer, because these formulations were colloidally stable in BSA buffer or showed at least significantly weaker aggregation (fluidMAG-Heparin, -OS). As discussed above for Resovist $^{\circledR}$, fluidMAG-D, -DP, (-DXS), -Heparin, and -OS this aggregation was rather caused by an agglutination of MNPs by FCS components like IgG antibodies, indicated by a concentration dependent maximum of aggregation. Note, that there is no obvious link of this aggregation behaviour to a special kind of functional groups, and no correlation to the charge of the MNPs in pure water. Here, we will mention, that the particle charges appear screened in CCM, shown by the reduced absolute value of zeta potential in CCM as well as by inversion of positive charge of DEAE and PEI (table 1). The charge inversion might be the result of attachment of negatively charged molecules, e.g. albumin, to the particle's surface. This picture of stabilisation of MNPs by proteins seems to hold also for the remarkably increased colloidal stability of dimercaptosuccinnic acid-stabilised MNPs in RPMI1640 medium caused by FCS observed by Chen et al. 2008. In the case of fluidMAG-CMX and -PGS, we did not observe such concentration dependence of aggregation. At least for fluidMAG-CMX this could be understood by the observation of strong aggregation (long relaxation times) already in CCM without FCS. This may be attributed to the much stronger effect to the screening of repulsive potential by higher valence ions (Sonntag\&Strenge 1987), present in CCM but not in BSA buffer. Looking at additional in vitro and in vivo investigations those MNPs have to be treated as careful as MNPs that are colloidally not stable in both BSA buffer and CCM (see above). With regard to the different aggregation behaviour in BSA buffer and CCM it is actually not possible to predict their activities in vivo. Therefore, further studies with fluidMAG-CMX, -PGS, -DP, -Heparin and -OS in whole blood as suspension medium have to be performed to assess the aggregation behaviour in vivo. 


\section{Conclusion}

The method of Magnetorelaxometry (MRX) allows the robust and sensitive quantification of aggregates of magnetic nanoparticles (MNPs) in different media, long before it becomes visible by precipitation. We found that strong visual aggregation correlates well with the aggregate size distribution parameter, derived from MRX data. Among the investigated samples MNPs coated with electrically neutral dextran (fluidMAG-DX) presented the highest colloidal stability followed by fluidMAG-ARA (polyarabic acid coating), targetMAG-NC (sodium phosphate) and fluidMAG-D (starch) which showed also good colloidal stability in cell culture medium (CCM). The other investigated MNP formulations with caboxymethyl dextran, polygalacturonic acid, phosphorylated starch, dextransulfate, heparin, oleic acid diethylaminoethyl and polyethylenimine as coating materials aggregated in CCM. Partially, this aggregation depended on the FCS concentration and was thus attributed to an agglutination of MNPs by serum components presumably $\operatorname{IgG}$ antibodies. The corresponding molecular target on the MNP surface remains to be investigated.

It seems likely that the uptake of magnetic nanoparticles depends on their size, thus the aggregation as a result of the interplay between coating of MNPs and the medium may determine the uptake significantly. As a consequence for further in vitro and in vivo investigations and moreover for clinical applications our results illustrate remarkably that the aggregation behaviour of iron oxide magnetic nanoparticles in physiological media depends on their coating and their concentration. Again, the necessity for biocompatibility studies with MNPs in biological environments for biomedical applications was emphasized.

\section{Acknowledgement}

The research was supported by BMBF Project Nanomagnetomedizin Grant No. FKZ-13N9150. 


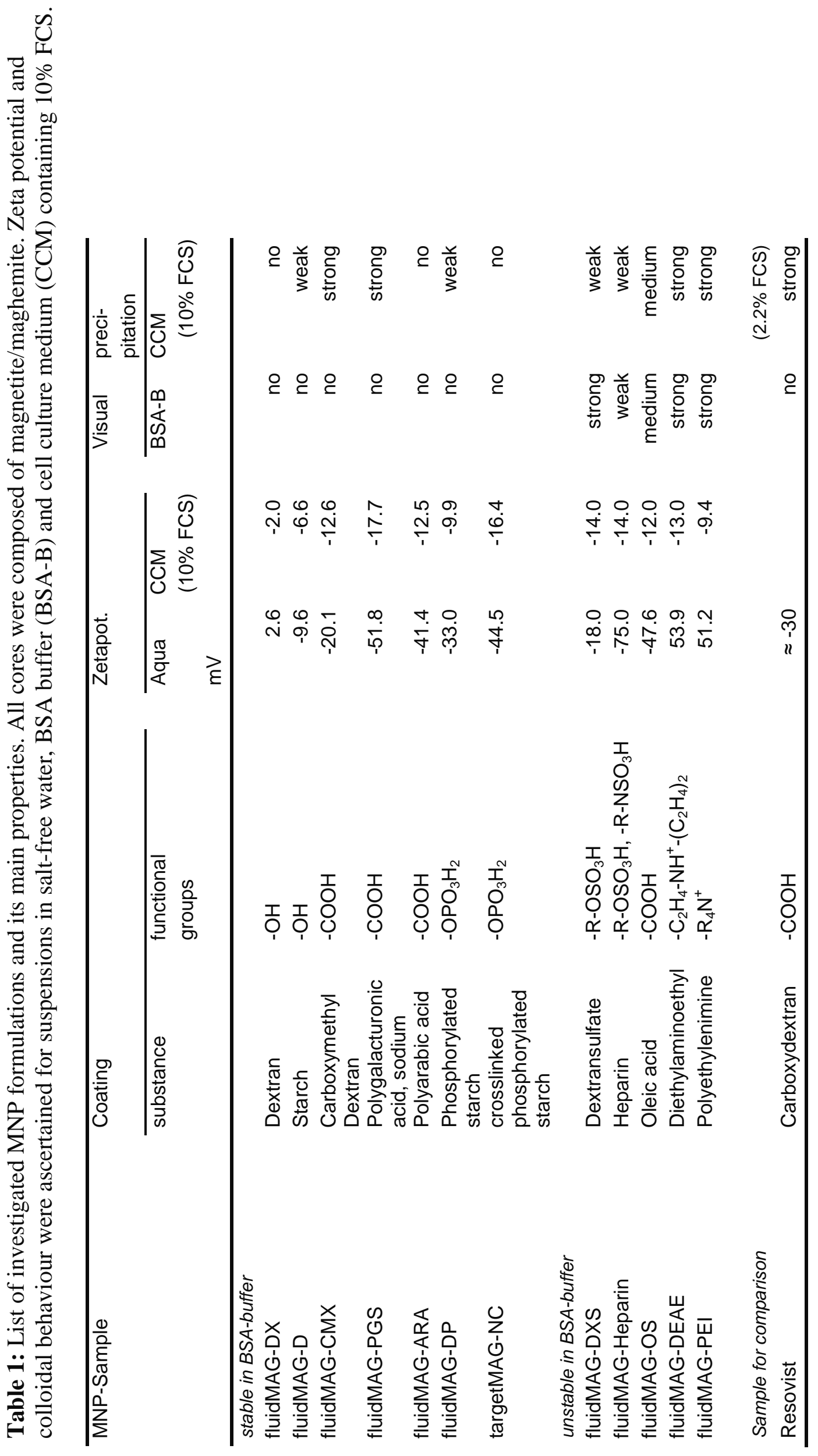




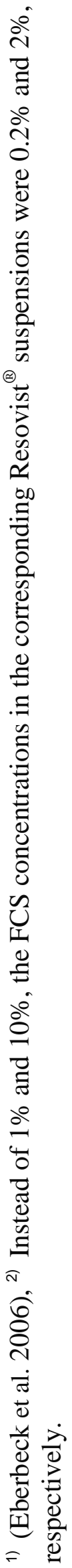




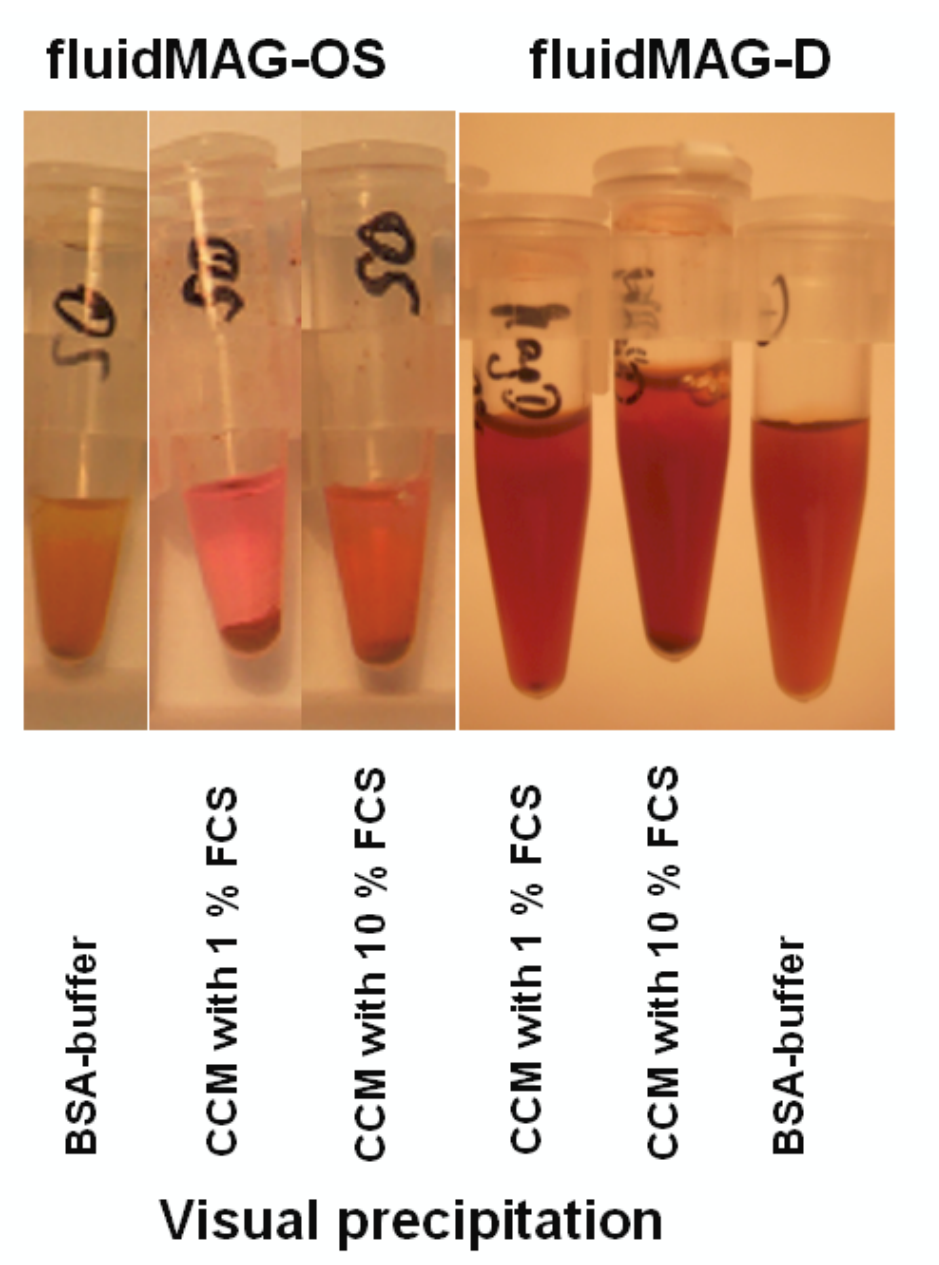

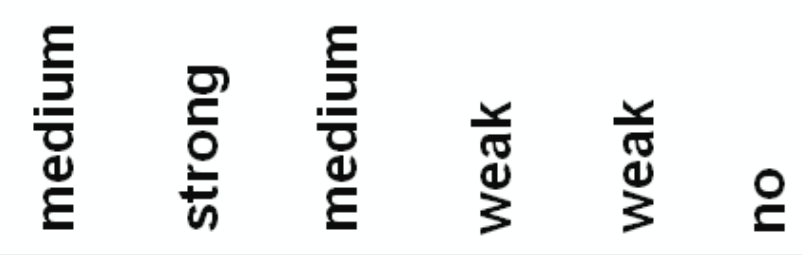

Figure 1: Examples of MNP suspensions 19 hours after preparation in different media as indicated. "FCS 1\%" and "FCS 10\%" refers to cell culture medium (CCM) containing $1 \%$ and $10 \%$ FCS, respectively. According to the quality of the visual appearance of the samples 4 classes of precipitation are defined: (i) no precipitation: the samples are homogeneously coloured; (ii) weak precipitation: A slight precipitate is observable and the supernatant remains homogeneously coloured like the related sample without precipitation (e.g. reference sample in BSA buffer); (iii) medium precipitation: A precipitate is observable and the supernatant is lighter coloured than that of the reference or the staining of the supernatant shows a gradient; (iv) strong precipitation: Almost all MNPs are precipitated, i.e. the supernatant is clear or at least much lighter coloured than the reference sample. Note that the CCM has a red colour determining the colour of supernatant of samples with strong aggregation in CCM. 


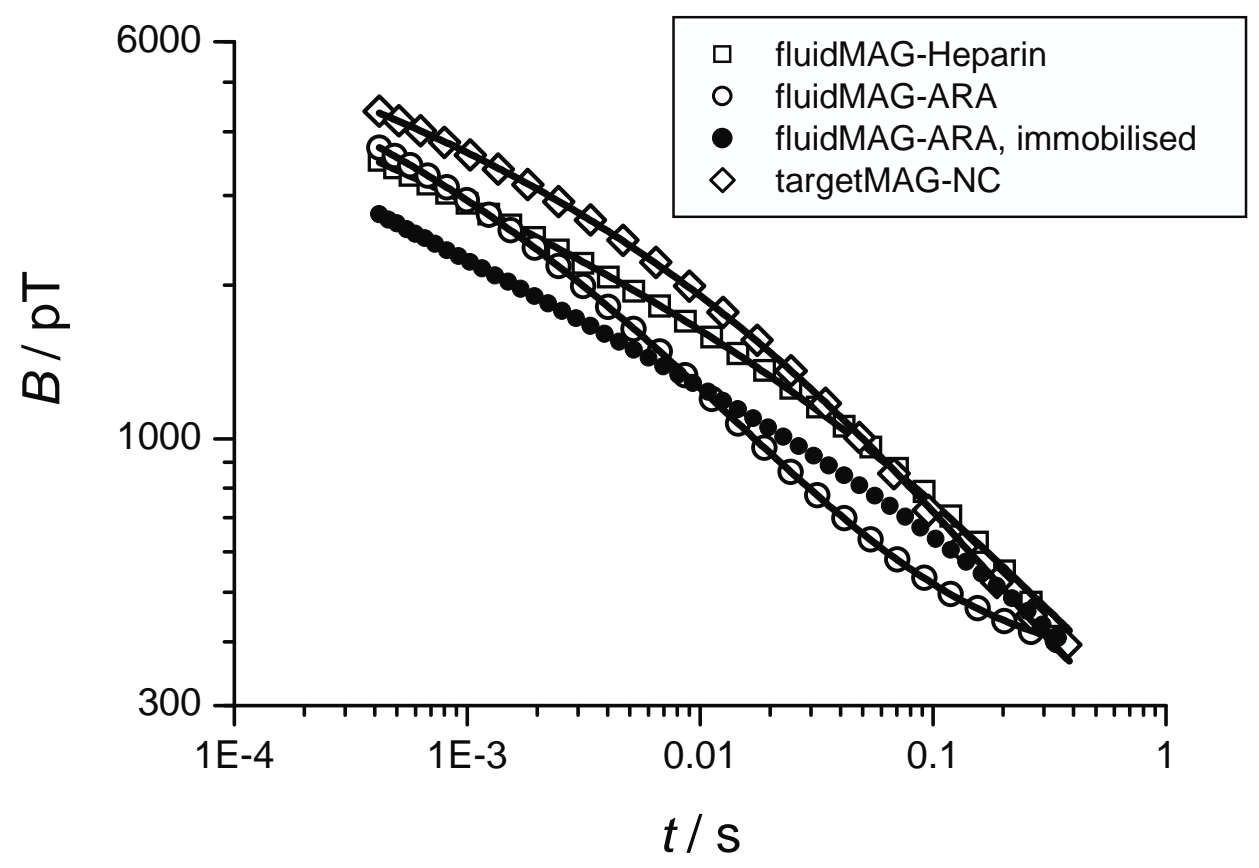

Figure 2: MRX curves for different MNP formulations suspended in 1\% FCS solution (open symbols). The lines represent the CMSM which was fitted to the data. The MRX curve of a freeze dried sample, where the MNPs were immobilised, was also depicted (filled symbols). The iron concentration of all samples was about $5 \mathrm{mmol} / \mathrm{l}$. The measurement was performed 90 min after preparation. For a better visual comparison of the line shapes, the baselines of the relaxation curves were adjusted (see text).

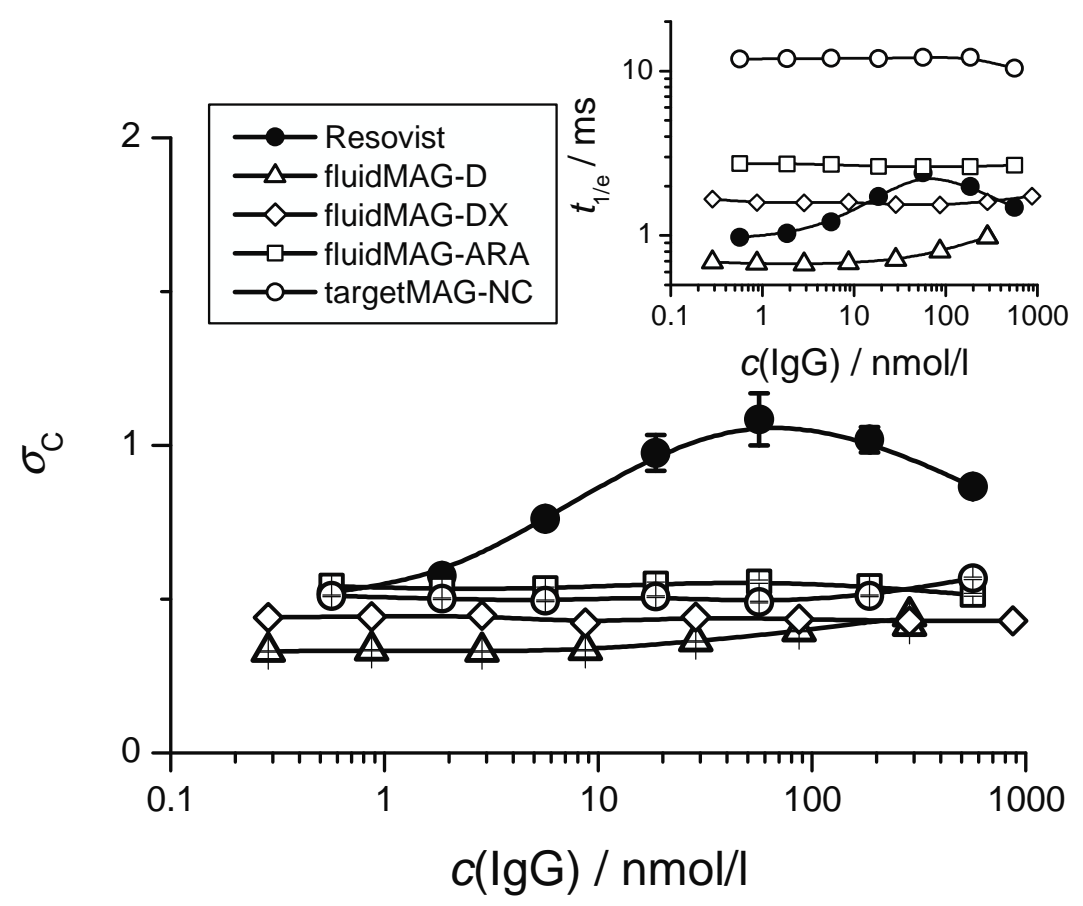

Figure 3: Geometric dispersion parameter $\sigma_{\mathrm{C}}$ of the cluster diameter distribution function obtained by fitting the CMSM to the MRX curves measured for samples with different IgG concentrations being related to the different FCS contents. The iron concentration of all samples was about $1.6 \mathrm{mmol} / \mathrm{l}$. Samples were measured about 3 hours after preparation. The lines serve as guide to the eye only. 


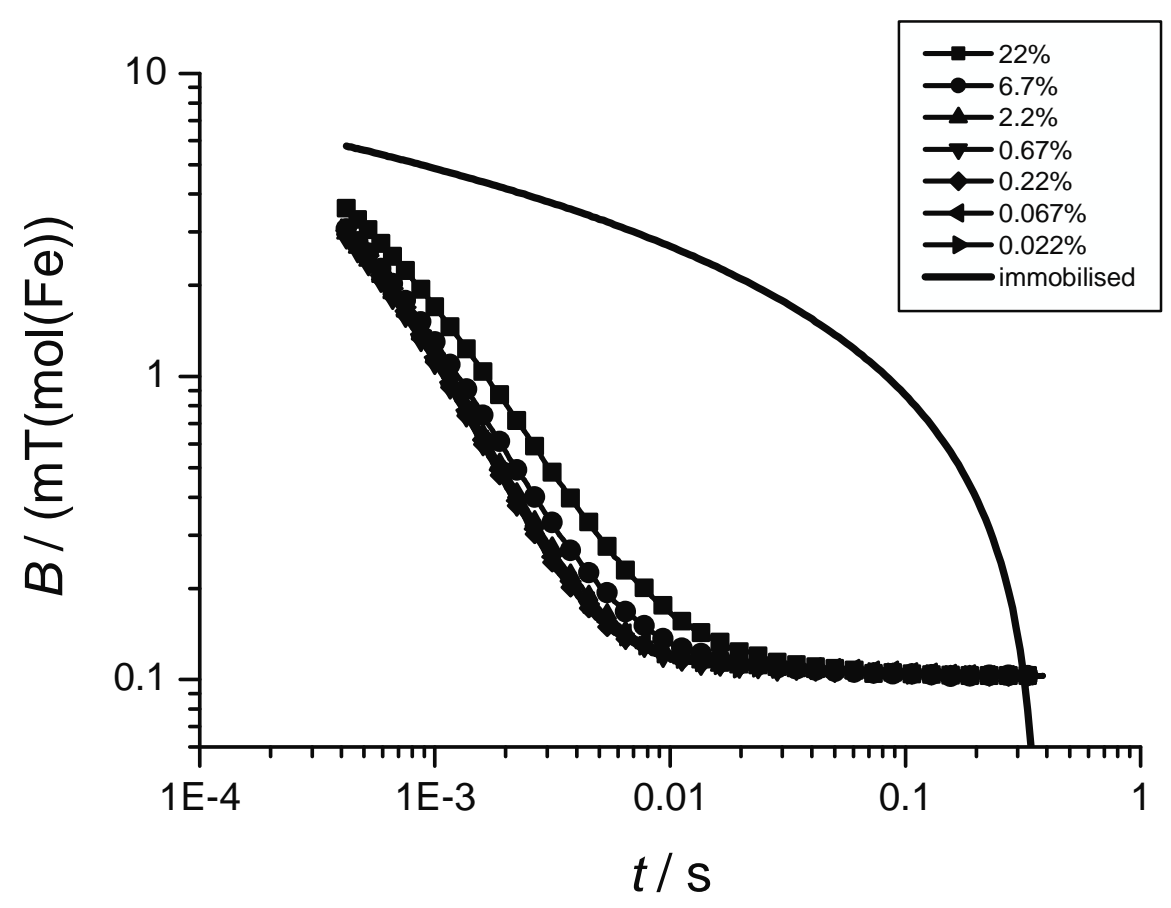

Figure 4: MRX curves for fluidMAG-D suspended in cell culture medium (CCM) with different FCS concentrations as indicated. For comparison, the MRX curve for immobilised fluidMAG-D was added. The iron concentration was $3.5 \mathrm{mmol} / \mathrm{l}$. The samples were measured 3 hours after preparation.

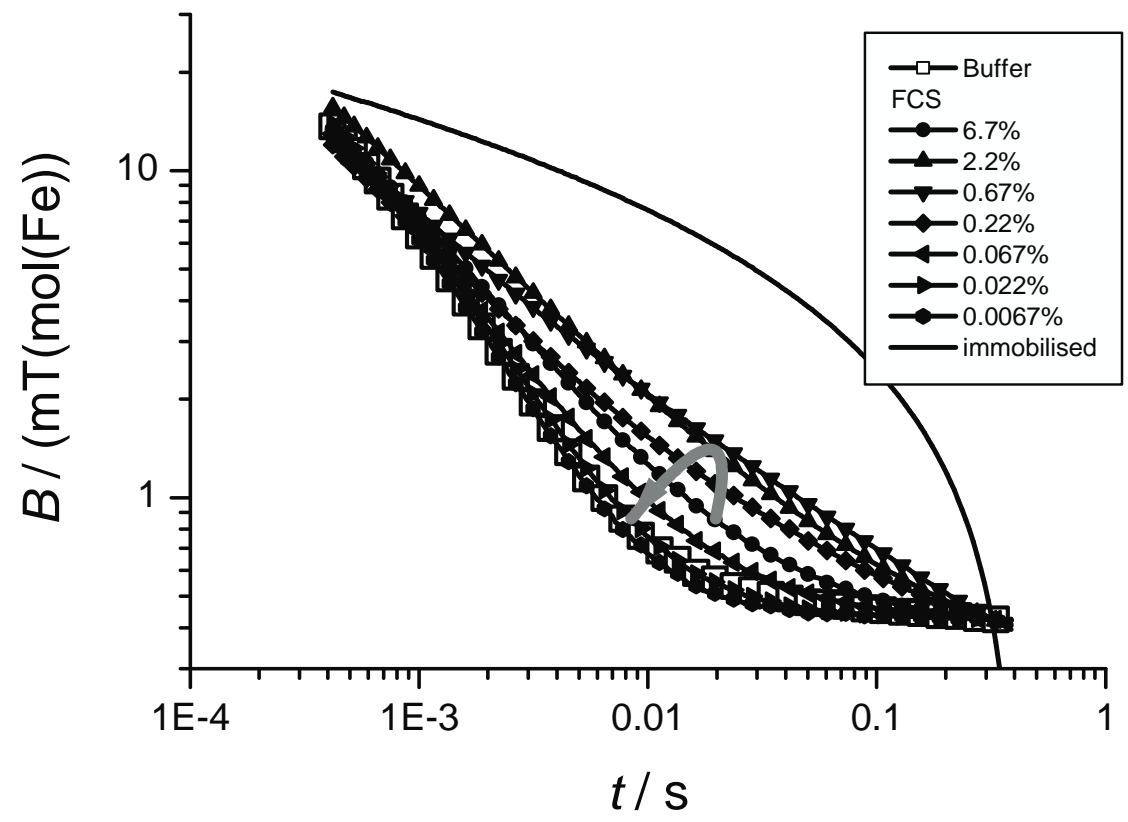

Figure 5: MRX curves for Resovist ${ }^{\circledR}$ suspended in cell culture medium (CCM) with different FCS concentrations as indicated. For comparison, the MRX curve for immobilised Resovist ${ }^{\circledR}$ was added. The gray arrow illustrates the development of the MRX curves for decreasing FCS concentration. The iron concentration was $1.7 \mathrm{mmol} / \mathrm{l}$. The samples were measured 3 hours after preparation. 


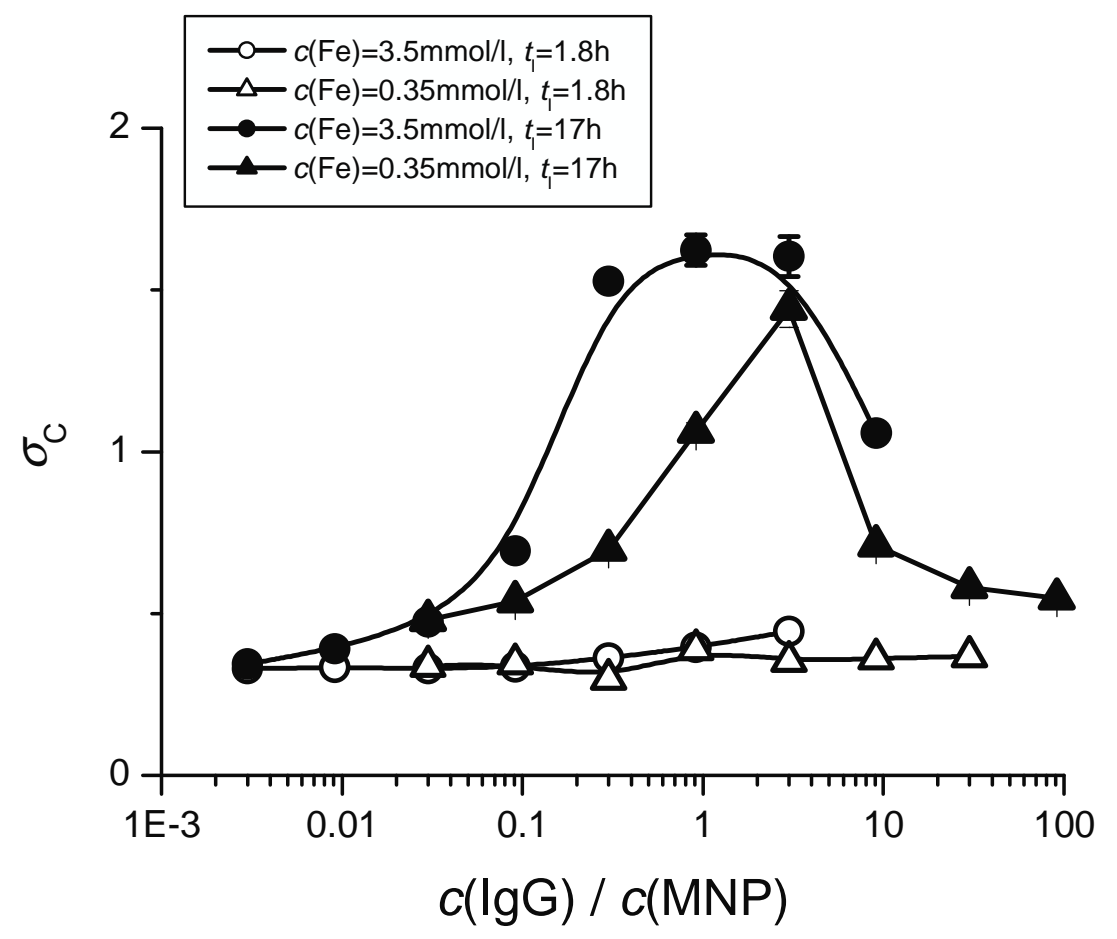

Figure 6: Representation of the geometric dispersion parameter $\sigma_{\mathrm{C}}$ of the aggregate size distribution function for fluidMAG-D suspended in FCS for different IgG-to-MNP concentration ratios, measured 1.8 and 17 hours after preparation. The lines serve as guide to the eye. 


\section{References}

Alexiou C, Jurgons R, Seliger C, Brunke O, Iro H and Odenbach S 2007 Delivery of superparamagnetic nanoparticles for local chemotherapy after intraarterial infusion and magnetic drug targeting Anticancer Res. 27 2019-22.

Bergemann C 1996 German Patent Application No. DE19624426.9.

Bruners P, Braunschweig T, Hodenius M, Pietsch H, Penzkofer T, Baumann M, Günther R W, Schmitz-Rode T and Mahnken A H 2009 Thermoablation of malignant kidney tumors using magnetic nanoparticles: an in vivo feasibility study in a rabbit model Cardiovasc. Intervent. Radiol.

Chen Z P, Zhang Y, Xu K, Xu R Z, Liu J W and Gu N 2008 Stability of hydrophilic magnetic nanoparticles under biologically relevant conditions J. Nanosci. and Nanotechnol. 8 6260-65

Chithrani B D, Ghazani A A and Chan W C 2006 Determining the size and shape dependence of gold nanoparticle uptake into mammalian cells Nano. Lett. 6 662-8.

Clement J H, Schwalbe M, Buske N, Wagner K, Schnabelrauch M, Görnert P, Kliche K O, Pachmann K, Weitschies W, Höffken K 2006 Differential interaction of magnetic nanoparticles with tumor cells and peripheral blood cells J. Cancer Res. Clin. Oncol. 132 287292.

Dass M, Landfester K and Walther P 2007 Cellular uptake of polymer nanoparticles imaged by electron microscopy based on high-pressure freezing Microscopy and Microanalysis 13 $220-1$.

Eberbeck D, Bergemann C, Wiekhorst F and Glöckl G 2005 Quantificationof aggregates of magnetic nanoparticles in different suspension media by magnetorelaxometry, Magnetohydrodynamics 41, 305-16.

Eberbeck D, Wiekhorst F, Steinhoff U and Trahms L 2006 Aggregation behaviour of magnetic nanoparticle suspensions investigated by magnetorelaxomery J. Phys.: Condens. matter 18 S2829-46.

Eberbeck D, Wiekhorst F, Steinhoff U, Schwarz K O, Kummrow A, Kammel M, Neukammer J and Trahms L 2009 Specific binding of magnetic nanoparticle probes to platelets in whole blood detected by magnetorelaxometry J. Magn. Magn. Mat. 321, 1617-20.

Gupta A K, Gupta M 2005 Synthesis and surface engineering of iron oxide nanoparticles for biomedical applications, Biomaterials. 26, 3995-4021.

Hergt R, Dutz S, Müller R, Zeisberger M 2006 J. Phys.: Condens. Matter. 18 S2919-34.

Hilger I, Andrä W, Hergt R, Hiergeist R, Schubert H, Kaiser W A 2001 Electromagnetic heating of breast tumors in interventional radiology: in vitro and in vivo studies in human cadavers and mice Radiology 218, 570-5.

Hilger I, Hergt R, Kaiser W A 2005 Use of magnetic nanoparticle heating in the treatment of breast cancer, IEE Proc. Nanobiotechnol. 152 33-9. 
Ito A, Tanaka K, Honda H, Abe S, Yamaguchi H, Kobayashi T 2003 Complete regression of mouse mammary carcinoma with a size greater than $15 \mathrm{~mm}$ by frequent repeated hyperthermia using magnetite nanoparticles J. Biosci. Bioeng. 96 364-9.

Kettering M, Winter J, Zeisberger M, Bremer-Streck S, Oehring H, Bergemann C, Alexiou C, Hergt R, Halbhuber K J, Kaiser W A and Hilger I 2007 Nanotechnology 18175101.

LaConte L, Nitin N and Bao G 2005 Magnetic nanoparticle probes Nanotoday 8 32-8.

Lawaczeck R, Bauer H, Frenzel T, Hasegawa M, Ito Y, Kito K, Miwa M, Tsutsui H, Vogler H and Weinmann H J 1997 Magnetic iron oxide nanoparticles coated with carboxydextran for parenteral administration and liver contrasting. Preclinical Profile of SH U555A Acta Radiologica 38 584-97.

Lin M M, Kim D K, El Haj A J, Dobson J 2008 Development of superparamagnetic iron oxide nanoparticles (SPIONS) for translation to clinical applications IEEE Trans. Nanobioscience 7 298-305.

Matz H, Drung D, Hartwig S, Groß H, Kötitz R, Müller W, Vass A, Weitschies W and Trahms L 1999 A SQUID measurement system for immunoassays Appl. Supercond. 6 57783.

Mykhaylyk O, Zelphati O, Hammerschmid E, Anton M, Rosenecker J, Plank C 2009 Recent advances in magnetofection and its potential to deliver siRNAs in vitro Methods Mol. Biol. 487 111-46.

Peng X H, Qian X, Mao H, Wang A Y, Chen Z G, Nie S, Shin D M 2008 Targeted magnetic iron oxide nanoparticles for tumor imaging and therapy Int. J. Nanomedicine 3 311-21.

Polyak B, Friedman G 2009 Magnetic targeting for site-specific drug delivery: applications and clinical potential Expert Opin. Drug Deliv. 6 53-70.

Sahoo S K and Labhasetwar V 2006 Nanoparticle Interface: An important determinant in nanoparticle-mediated drug/gene delivery Nanoparticle Technology for Drug Delivery ed R B Gupta and U B Kompella (Taylor and Francis) 148

Sonntag H and Strenge K 1987 Coagulation Kinetics and Structure Formation (New York. Plenum Press).

Sosnovik D E, Nahrendorf M and Weissleder R 2008 Magnetic nanoparticles for MR imaging: agents, techniques and cardiovascular applications Basic Res. Cardiol. 103 122-30.

TiefenhauerL X, Tschirky A, Kühne G and Andres R Y 1996 In vivo evaluation of magnetite nanoparticles for use as a tumor contrast agent in MRI Magnetic Resonace Imaging 14 391402.

Thiesen B and Jordan A 2008 Clinical applications of magnetic nanoparticles for hyperthermia Int. J. Hyperthermia 24 467-74. 
Wilhelm C, Fortin J P and Gazeau F 2007 Tumor cell toxicity of intracellular hyperthermia mediated by magnetic nanoparticles J. Nanosci. Nanotechnol. 7 2933-37.

Win K Y and Feng S S. Effects of particle size and surface coating on cellular uptake of polymeric nanoparticles for oral delivery of anticancer drugs Biomaterials 26, 2713-22.

Zhang S, Li J, Lykotrafitis G, Bao G and Suresh S 2009 Size-Dependent Endocytosis of Nanoparticles., Adv Mater Deerfield 21 419-24.

Zhang Y, Kohler N and Zhang M 2002 Surface modification of superparamagnetic nagnetite nanoparticles and their intracellular uptake, Biomaterials 23, 1553-61. 